\title{
Registered Nurse Practitioner
}

National Cancer Institute

\section{Source}

National Cancer Institute. Registered Nurse Practitioner. NCI Thesaurus. Code C71279.

A registered nurse who has received special training and can perform many of the duties of a physician. 